\title{
Method Development and Validation of Darifenacin in Rat Plasma by Liquid Chromatography-Tandem Mass Spectrometry: Application to a Pharmacokinetic Study
}

\author{
Thejomoorthy Karavadi ${ }^{1}$ and B. R. Challa ${ }^{2}$ \\ ${ }^{1}$ Pacific University of Higher Education and Research, Udaipur, Rajasthan 313001, India \\ ${ }^{2}$ Department of Pharmaceutical Chemistry, Nirmala College of Pharmacy, Madras Road, Kadapa 516002, Andhra Pradesh, India
}

Correspondence should be addressed to B. R. Challa, baluchalla_99@yahoo.com

Received 12 August 2012; Accepted 4 October 2012

Academic Editors: F.-F. Hsu, E. Saint-Aman, and E. Stathatos

Copyright ( $) 2012$ T. Karavadi and B. R. Challa. This is an open access article distributed under the Creative Commons Attribution License, which permits unrestricted use, distribution, and reproduction in any medium, provided the original work is properly cited.

A selective, sensitive, and high-throughput liquid chromatography-tandem mass spectrometry (LC-ESI-MS/MS) method has been developed and validated for the quantitation of darifenacin in rat plasma. Sample clean up involved liquid-liquid extraction (LLE) and used $100 \mu \mathrm{L}$ of rat plasma. Zorbax, SB C18, $4.6 \times 75 \mathrm{~mm}, 3.5 \mu \mathrm{m}$ particle size analytical column using $10 \mathrm{mM}$ ammonium acetate buffer $(\mathrm{pH} 5)$ and methanol $(10: 90, \mathrm{v} / \mathrm{v})$ as the mobile phase was used. The parent $\rightarrow$ product ion transitions for the $\operatorname{drug}(m / z 427.3 \rightarrow 147.3)$ and IS $(m / z 431.4 \rightarrow 151.2)$ were monitored on a triple quadrupole mass spectrometer, operating in the multiple reaction monitoring (MRM) and positive ion mode. The method was validated over the concentration range of 10.00 $20000.00 \mathrm{pg} / \mathrm{mL}$ for darifenacin. The method was successfully applied into a pharmacokinetic study in rat plasma under fasting conditions.

\section{Introduction}

Darifenacin hydrobromide is $(S)-2-\{1-[2-(2,3$-dihydrobenzofuran-5-yl)ethyl]-3-pyrrolidinyl $\}$-2,2-diphenylacetamidehydrobromide. Its empirical formula is $\mathrm{C}_{28} \mathrm{H}_{30} \mathrm{~N}_{2} \mathrm{O}_{2} \cdot \mathrm{HBr}$ with molecular weight 507.47 (Figure 1). Darifenacin is used to treat urinary incontinence. The recommended starting dose of darifenacin extended-release tablets is $7.5 \mathrm{mg}$ once daily. Based upon an individual response, the dose may be increased to $15 \mathrm{mg}$ once daily, as early as two weeks after starting therapy. Darifenacin is approximately $98 \%$ bound to plasma proteins and metabolized by the liver. Metabolism is mediated by cytochrome P450 enzymes CYP2D6 and CYP3A4. Elimination is $60 \%$ by urine and $40 \%$ through feces. The elimination half-life of darifenacin is approximately $13-19$ hours [1-5].

Literature survey reveals that there are few methods reported for quantitation and identification of darifenacin in pharmaceutical formulations [6-15] and biological matrices [16-20]. These methods are developed with different analytical instruments like UV $[7,8]$, spectroflurimetric [9], HPTLC [10], HPLC [11-14], LC-MS [15, 19, 20], and positron emission tomography [16]. Among all the reported methods quantification in rats [16], mice [18], and human plasma $[19,20]$ methods are helpful for clinical pharmacokinetics. The reported methods $[16,18-20]$ do not show the accurate and rugged method. It is required to develop and validate the most economical, simple, rugged, and reproducible bioanalytical method for quantification of darifenacin in biological matrices for its clinical pharmacokinetics.

The objective of the present study is to develop a simple, sensitive, selective, rapid, rugged, and reproducible method by using LC-ESI-MS/MS method for quantitation in rat plasma.

\section{Experimental}

2.1. Chemicals and Materials. Reference standards of darifenacin $(99.7 \%)$ and darifenacin $\mathrm{d} 4(95.3 \%$, IS) were procured 


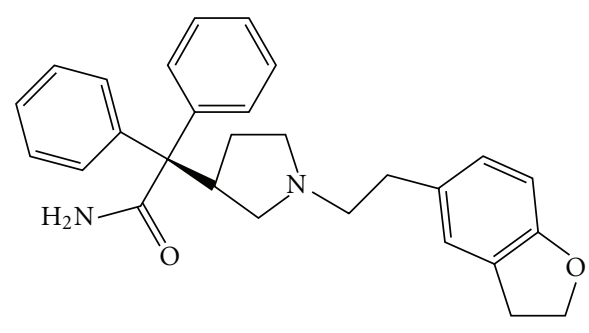

Darifenacin

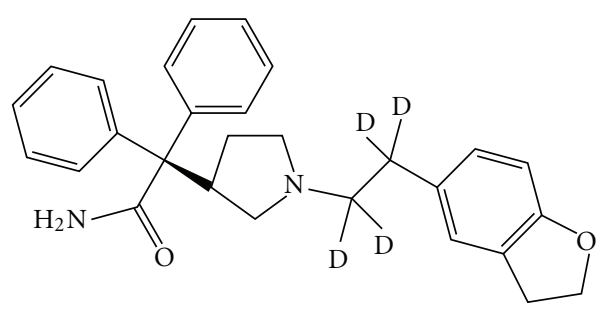

Darifenacin-d4

FIgURE 1: Chemical structures of darifenacin and darifenacin-d4.

from TLC Pharmachem (Canada). HPLC grade methanol was obtained from Mallinckrodt Baker, (S.A. de C.V., Mexico). Reagent grade ammonium acetate and sodium hydroxide were obtained from Merck Specialties Pvt. Ltd., (Mumbai, India). HPLC grade methyl tertiary butyl ether and dichloromethane were obtained from RCI Labscan, Mumbai. Water used in the entire analysis was prepared from Milli-Q water purification system procured from Millipore (Bangalore, India). Blank rat plasma was procured from Bioneeds (Bangalore, India) and was stored at $-30^{\circ} \mathrm{C}$ until use.

2.2. Instrumentation. The 1200 Series HPLC system (Agilent Technologies, Waldbronn, Germany) connected to the API 4000 triple quadrupole mass spectrometer (ABI-SCIEX, Toronto, Canada) with a turbo Electrospray interface in positive-ion mode was used for detection. Data processing was performed on Analyst 1.4.1 software package (SCIEX).

2.3. Standard Stock, Calibration Standards, and Quality Control Sample Preparation. The standard stock solution of $50 \mu \mathrm{g} / \mathrm{mL}$ of darifenacin was prepared by dissolving requisite amount in methanol. Calibration standards and quality control (QC) samples were prepared by spiking ( $2 \%$ of total volume of blank plasma) blank plasma with stock solution. Calibration curve standards were made at 10.00 , $20.00,500.00,1000.00,2000.00,4000.00,8000.00,12000.00$, 16000.00 , and $20000.00 \mathrm{pg} / \mathrm{mL}$ while quality control samples were prepared at four levels, namely, $18000.00 \mathrm{pg} / \mathrm{mL}$ (HQC, high quality control), $9000.00 \mathrm{pg} / \mathrm{mL}$ (MQC, middle quality control), $30.00 \mathrm{pg} / \mathrm{mL}$ (LQC, low quality control), and $10.00 \mathrm{pg} / \mathrm{mL}$ (LLOQC, lower limit of quality control) for darifenacin (see Table 1 and Figure 4).

Stock solution of the internal standard (darifenacind4) was prepared by dissolving appropriate amount of darifenacin-d 4 in $25 \mathrm{~mL}$ of methanol to obtain $50 \mu \mathrm{g} / \mathrm{mL}$ stock solution (Figure 1). An aliquot of $500 \mu \mathrm{L}$ of this solution was further diluted to $50.0 \mathrm{~mL}$ in the same diluent. From this intermediate dilution $2 \mathrm{~mL}$ was spiked into $50.0 \mathrm{~mL}$ volumetric flask and diluted with $90: 10 \mathrm{v} / \mathrm{v}$ methanol: $10 \mathrm{mM}$ ammonium acetate to obtain a solution of $20 \mathrm{ng} / \mathrm{mL}$. Standard stock solution and IS dilution, aqueous standards were stored at $2-8^{\circ} \mathrm{C}$ in the refrigerator. Calibration standards and quality control samples were stored at $-30^{\circ} \mathrm{C}$ until use.
TABLE 1: Calibration curve details.

\begin{tabular}{lccc}
\hline $\begin{array}{l}\text { Concentration } \\
(\mathrm{pg} / \mathrm{mL})\end{array}$ & Mean $\pm \mathrm{SD}$ & $\% \mathrm{CV}$ & Accuracy \\
\hline 10.00 & $9.80 \pm 0.08$ & 0.79 & 98.01 \\
20.00 & $20.86 \pm 0.34$ & 1.65 & 104.30 \\
500.00 & $484.02 \pm 11.21$ & 2.32 & 96.80 \\
1000.00 & $992.82 \pm 12.35$ & 1.24 & 99.28 \\
2000.00 & $2000.80 \pm 33.79$ & 1.69 & 100.04 \\
4000.00 & $4074.72 \pm 33.03$ & 0.81 & 101.87 \\
8000.00 & $8149.69 \pm 121.44$ & 1.49 & 101.87 \\
12000.00 & $11936.52 \pm 209.10$ & 1.75 & 99.47 \\
16000.00 & $15974.45 \pm 290.69$ & 1.82 & 99.84 \\
20000.00 & $19968.06 \pm 363.36$ & 1.82 & 99.84 \\
\hline
\end{tabular}

2.4. Chromatographic Condition. Chromatographic separation was carried out on a reversed phase Zorbax, SB C18, $4.6 \times 75 \mathrm{~mm}, 3.5 \mu \mathrm{m}$ column using at $40^{\circ} \mathrm{C}$ with mixture of $10 \mathrm{mM}$ ammonium acetate buffer $(\mathrm{pH} 5)$ and methanol $(10: 90 \mathrm{v} / \mathrm{v})$ as mobile phase at a flow-rate of $1.0 \mathrm{~mL} / \mathrm{min}$. Retention time of darifenacin and darifenacin- $\mathrm{d} 4$ was found to be approximately $1.3 \pm 0.2 \mathrm{~min}$ for both drug and IS with a total runtime of 3 minutes.

2.5. Mass Spectrometric Conditions. Ionization and detection of analyte and IS were carried out on a triple quadrupole mass spectrometer, AB-SCIEX, (Toronto, Canada) equipped with Electrospray ionization and operating in positive ion mode. Quantitation was performed using multiple reaction monitoring (MRM) mode to monitor parent $\rightarrow$ product ion $(\mathrm{m} / \mathrm{z})$ transition for darifenacin was $427.3 \rightarrow$ 147.3 (Figures 2(a) and 2(b)). For internal standard, the $\mathrm{MH}^{+}(\mathrm{m} / \mathrm{z}:$ 431.4) was monitored as the precursor ion (Figure 3(a)) and a fragment at $\mathrm{m} / z$ : 151.2 was monitored as the product ion (Figure 3(b)). Mass parameters were optimised as source temperature $600^{\circ} \mathrm{C}$, nebulizer gas 30 (nitrogen) psi, heater gas 25 (nitrogen) psi, curtain gas 20 (nitrogen) psi, CAD gas 3 (nitrogen) psi, ion spray (IS) voltage 5500 volts, source flow rate $1 \mathrm{~mL} / \mathrm{min}$ without split, entrance potential $10 \mathrm{~V}$, declustering potential $40 \mathrm{~V}$ for both analyte and IS, collision energy $16 \mathrm{~V}$ for both analyte and IS, 


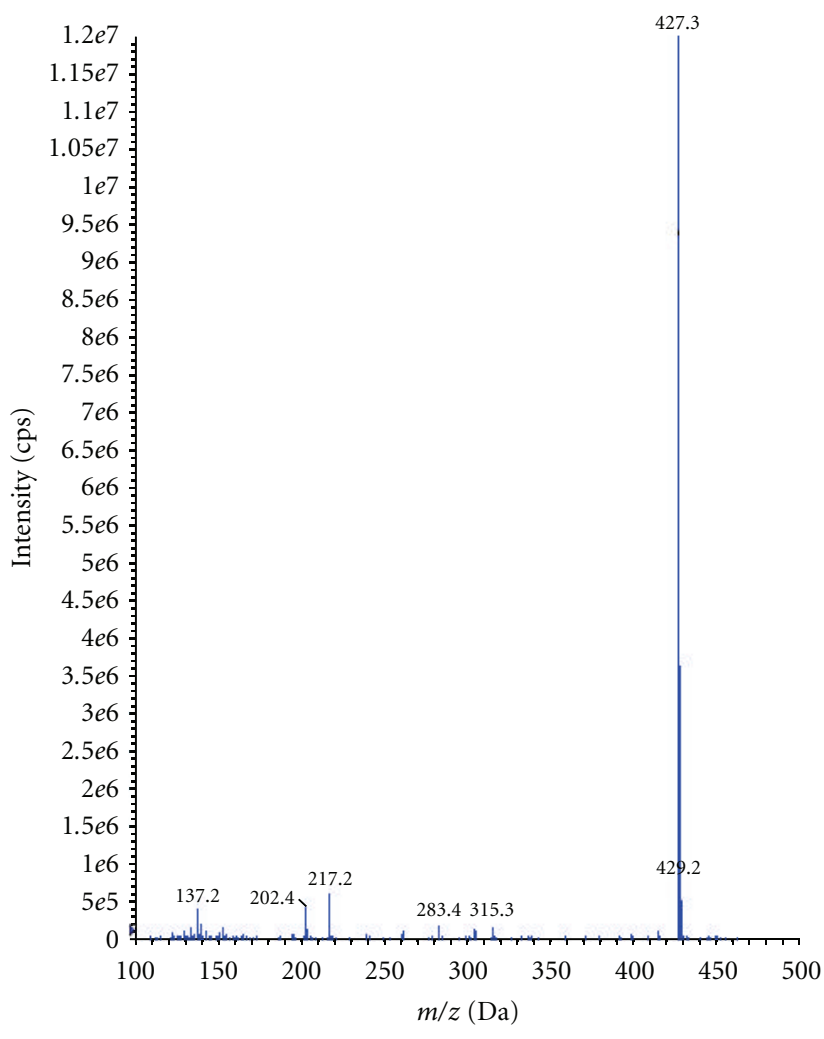

(a)

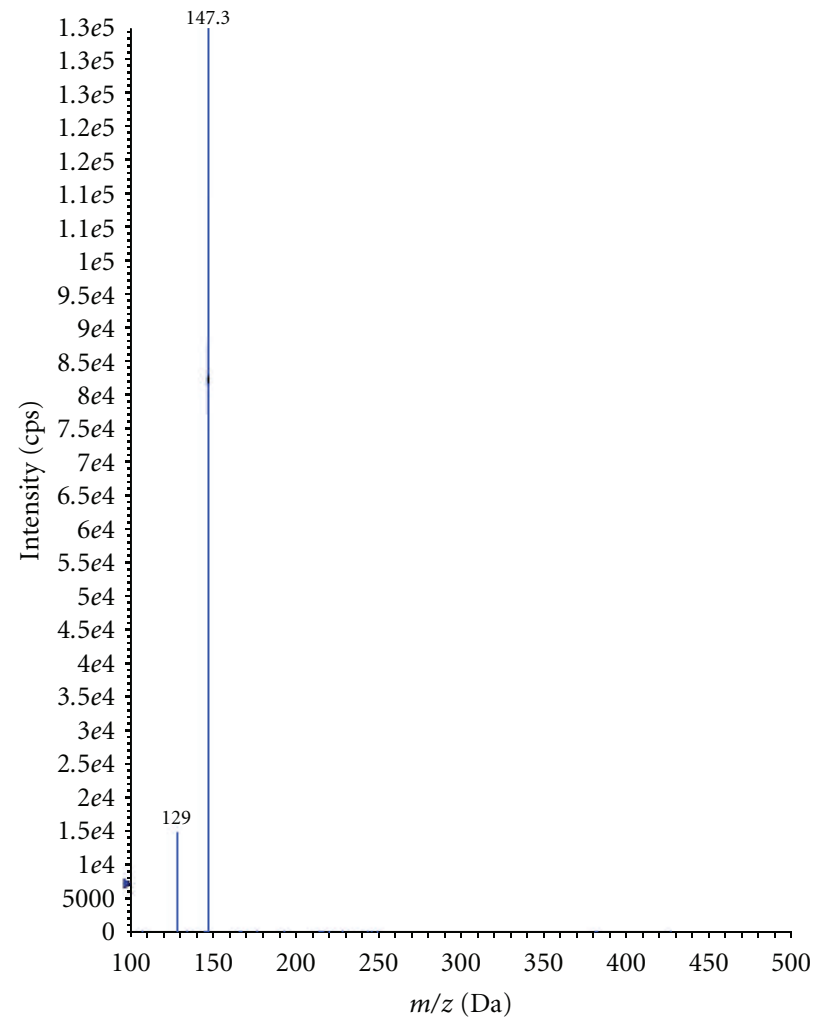

(b)

Figure 2: (a) ESI mass spectrum of darifenacin. (b) Product ion spectrum of the $[\mathrm{M}+\mathrm{H}]^{+}$ion of darifenacin.

collision cell exit potential $6 \mathrm{~V}$, and the dwell time was set at $200 \mathrm{~ms}$ for analyte and $8 \mathrm{~V}$ for IS.

2.6. Sample Preparation. Prior to analysis, all frozen subject samples, calibration standards, and quality control samples were thawed and allowed to equilibrate at room temperature. To an aliquot of $100 \mu \mathrm{L}$ of spiked plasma sample, $50 \mu \mathrm{L}$ internal standard was added and vortexed briefly. Further, $100 \mu \mathrm{L}$ of sodium hydroxide solution was added and vortexed briefly. To these samples, $2.5 \mathrm{~mL}$ of extraction solvent (methyl tertiary butyl ether: dichloromethane; $80: 20$, v/v) was added, capped, and the samples were vortexed for $15 \mathrm{~min}$. Centrifugation of the samples was done at $4000 \times \mathrm{g}$, for $5 \mathrm{~min}$ at $20^{\circ} \mathrm{C}$. Supernatant from each sample was transferred into respective tube and evaporated to dryness under nitrogen at $40 \pm 2{ }^{\circ} \mathrm{C}$. The dried samples were reconstituted with $200 \mu \mathrm{L}$ of methanol: $10 \mathrm{mM}$ ammonium acetate $(90: 10)$. All the tubes containing samples were vortexed briefly and transferred into autosampler vials for injection in the chromatographic system.

2.7. Method Validation. The method validation was performed as per the USFDA guidelines [21, 22]. System suitability experiment was performed by injecting three consecutive injections using aqueous solution of darifenacin and internal standard at the start of each batch during the method validation. The carryover effect of the autosampler was evaluated by injecting a sequence of injection solutions of aqueous standard, mobile phase, standard blank, and extracted standard equivalent to the highest standard in the calibration range. As per the acceptance criteria, the response in the blank should not be greater than $20 \%$ of LLOQ response.

The linearity of the method was determined by analysis of five linear curves containing ten non-zero concentrations. The ratio of area response for analyte to IS was used for regression analysis. Each calibration curve was analyzed individually by using least square weighted $\left(1 / x^{2}\right)$ linear regression. The lowest standard on the calibration curve was accepted as the lower limit of quantitation (LLOQ), if the analyte response was at least five times more than that of drug free (blank) extracted plasma. The deviation of standards other than LLOQ from the nominal concentration should not be more than $\pm 15.0 \%$. For LLOQ it should not be more than $\pm 20.0 \%$.

The selectivity of the method towards endogenous plasma matrix components was assessed in ten lots (5 normal of K2 EDTA plasma, 2 haemolysed, 2 lipimic, and 1 heparinised) of blank rat plasma. This was done to estimate the extent to which endogenous plasma components contribute towards interference at the retention time of analytes and IS. The cross talk of MRM for analytes and IS was checked using the highest standard on calibration curve and working solution of IS. 


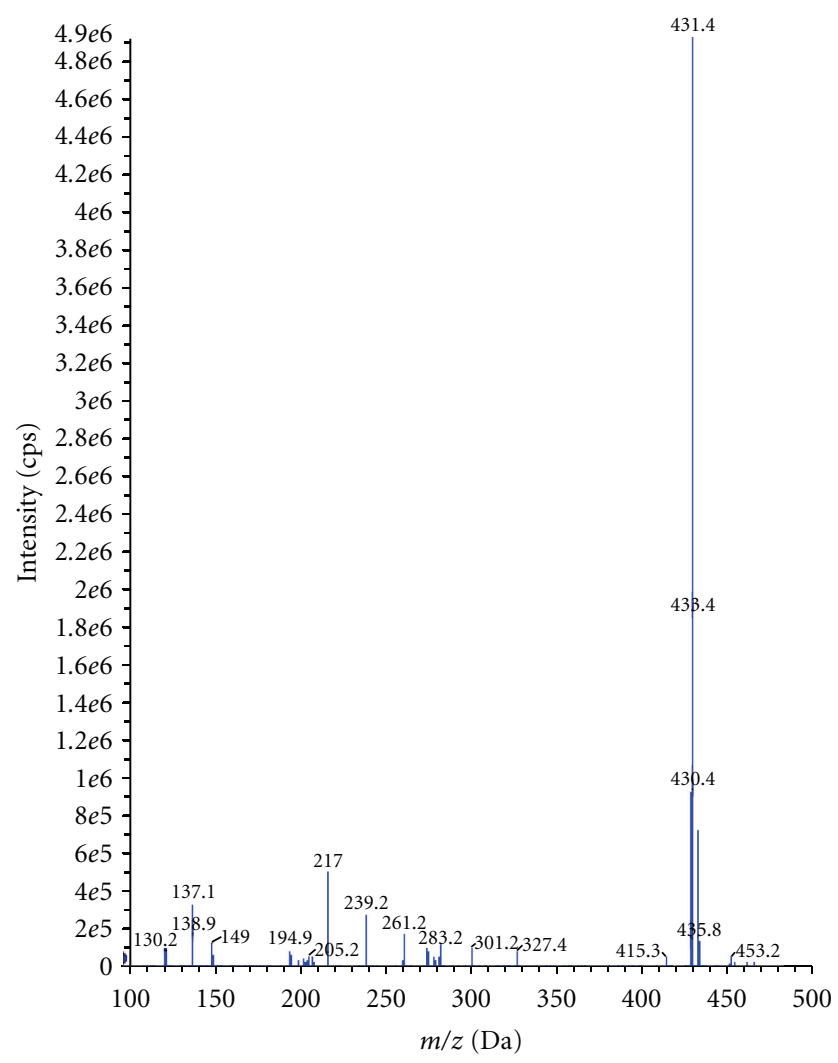

(a)

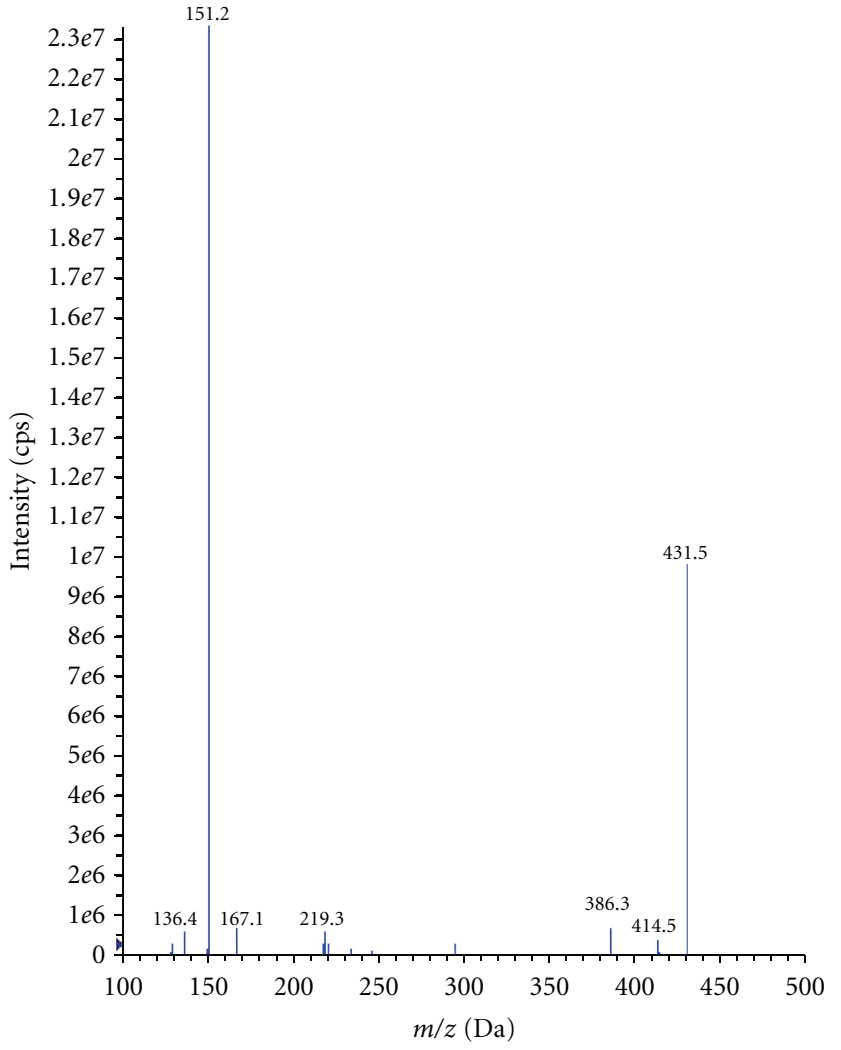

(b)

FIgURE 3: (a) ESI mass spectrum of darifenacin-d4. (b) Product ion spectrum of the $[\mathrm{M}+\mathrm{H}]^{+}$ion of darifenacin $\mathrm{d} 4$.

For determining the intra-day accuracy and precision, replicate analysis of plasma samples of darifenacin was performed on the same day. The run consisted of a calibration curve and six replicates of LLOQ, LQC, MQC, and HQC samples. The inter-day accuracy and precision were assessed by analysis of five precision and accuracy batches on four consecutive validation days. The precision of the method was determined by calculating the percent coefficient of variation $(\% \mathrm{CV})$ for each level. The deviation at each concentration level from the nominal concentration was expected to be within $\pm 15.0 \%$ except LLOQ, for which it should be within $\pm 20.0 \%$.

Ion suppression/enhancement effects on the MRM LCMS/MS sensitivity were evaluated by the post column analyte infusion experiment. A standard solution containing darifenacin (at MQC level) and IS was infused post column into the mobile phase at $10 \mu \mathrm{L} / \mathrm{min}$ employing the in-built infusion pump. Aliquots of $10 \mu \mathrm{L}$ of extracted control plasma were then injected into the column by the autosampler and MRM LC-MS/MS chromatogram was acquired for the analytes and IS. Any dip in the baseline upon injection of double blank plasma (without IS) would indicate ion suppression, while a peak at the retention time of analyte and IS indicates ion enhancement.

The relative recovery, matrix effect, and process efficiency were assessed; all three parameters were evaluated at HQC, MQC, and LQC levels in six replicates.
Relative recovery (RE) was calculated by comparing the mean area response of extracted samples (spiked before extraction) to that of unextracted samples (spiked after extraction) at each QC level. The recovery of IS was similarly estimated.

Absolute matrix effect (ME) was assessed by comparing the mean area response of unextracted samples (spiked after extraction) with mean area of neat standard solutions.

The overall "process efficiency" (\%PE) was calculated as $(\mathrm{ME} \times \mathrm{RE}) / 100$. The assessment of relative matrix effect was based on the direct comparison of the MS/MS responses (peak areas) of the analytes spiked into extracts originating from different lots of plasma. The variability in these responses, expressed as \%CV was considered as the measure of relative matrix effect.

Stability experiments were carried out to examine the analyte stability in stock solutions and in plasma samples under different conditions. Short-term stability at room temperature and long-term stability of spiked solution stored at $-30^{\circ} \mathrm{C}$ was assessed by comparing the area response of stability sample of analyte and IS with the area response of sample prepared from fresh stock solutions. The solutions were considered stable if the deviation from nominal value was within $\pm 10 \%$.

Autosampler (wet extract) stability, bench top stability, freeze-thaw stability, and long-term stability were performed at LQC and HQC, using six replicates at each level. The 

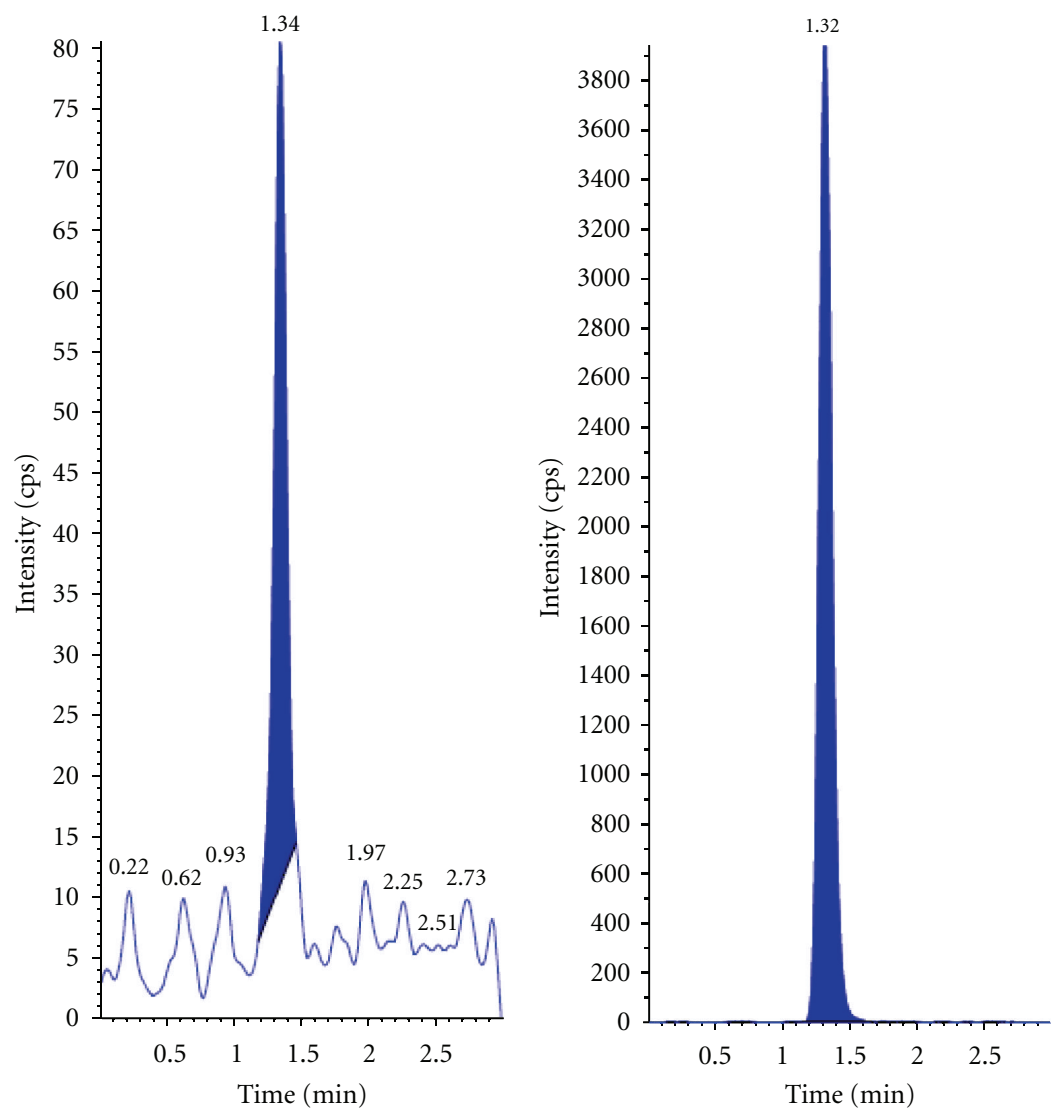

FIGURE 4: LLOQ chromatogram of darifenacin and darifenacin $\mathrm{d} 4$ in rat plasma.

samples were considered stable if the deviation from the mean calculated concentration of freshly thawed quality control samples was within $\pm 15 \%$.

To authenticate the ruggedness of the proposed method, it was done on two precision and accuracy batches. The first batch was analyzed by two different analysts while the second batch was analyzed on a different column.

Dilution integrity experiment was conducted by diluting the stock solution prepared as spiked standard at concentration of $30000.00 \mathrm{pg} / \mathrm{mL}$ for darifenacin. The precision and accuracy for dilution integrity standards at $1 / 4$ th $(7500.00 \mathrm{pg} / \mathrm{mL})$ and $1 / 10 \mathrm{th}(3400.00 \mathrm{pg} / \mathrm{mL})$ dilutions for darifenacin were determined by analyzing the samples against calibration curve standards.

2.8. Study Design. The validated method has been successfully used to quantify darifenacin concentrations in rat plasma. The study was conducted according to current GCP guidelines. Before conducting the study it was also approved by an authorized animal ethics committee. There was a total of 9 blood collection time points including the predose sample. The blood samples were collected in separate vacutainers containing $\mathrm{K}_{2}$ EDTA as anticoagulant. The plasma from these samples was separated by centrifugation at $3000 \mathrm{rpm}$ within the range of $2-8^{\circ} \mathrm{C}$. The plasma samples thus obtained were stored at $-30^{\circ} \mathrm{C}$ until analysis. Post analyses of the pharmacokinetic parameters were computed using WinNonlin software version 5.2.

\section{Results and Discussion}

3.1. Method Development. During method development, different options were evaluated to optimize mass spectrometry detection parameters, chromatography, and sample extraction.

\subsection{Mass Spectrometry Detection Parameter Optimization.} Electrospray ionization (ESI) provided a maximum response over atmospheric pressure chemical ionization (APCI) mode and was chosen for this method. The instrument was optimized to obtain sensitivity and signal stability during infusion of the analyte in the continuous flow of mobile phase to Electrospray ion source operated at both polarities at a flow rate of $10 \mu \mathrm{L} / \mathrm{min}$. Darifenacin gave more responses in positive ion mode as compare to the negative ion mode. The collisionally activated dissociation (CAD) mass spectrum of darifenacin shows formation of characteristic product ions at $m / z 129.0$ and 147.3. The major product ion at $m / z 147.3$ for darifenacin could be from the protonated precursor molecule. The CAD mass spectrum of darifenacin$\mathrm{d} 4$ shows formation of characteristic product ions at $\mathrm{m} / \mathrm{z}$ $136.4,151.2,167.1,219.3,386.3,414.5$, and 431.5. The major 
product ion at $\mathrm{m} / \mathrm{z} 151.2$ arose from the protonated precursor molecule. The predominant peaks in the primary ESI spectra of darifenacin and darifenacin-d4 corresponds to the $[\mathrm{M}+\mathrm{H}]^{+}$ions at $\mathrm{m} / z 427.3$ and 431.4 , respectively (Figures 2(a) and 2(b)). Product ions of darifenacin and darifenacin-d 4 were $\mathrm{m} / \mathrm{z}$ of 147.2 and 151.2 , respectively (Figures 2(a) and 2(b)).

3.3. Chromatography Optimization. Initially, a mobile phase consisting of ammonium formate and acetonitrile in varying combinations was tried, but a low response was observed. The mobile phase containing acetic acid:acetonitrile $(20: 80 \mathrm{v} / \mathrm{v})$ and acetic acid: methanol $(20: 80 \mathrm{v} / \mathrm{v})$ gives the better response, but poor peak shape was observed. A mobile phase with various strengths of ammonium formate in water at $\mathrm{pH} 5$ combination with methanol and acetonitrile with varying combinations were tried. Using a mobile phase containing ammonium formate in water in combination with methanol $(10: 90 \mathrm{v} / \mathrm{v})$, the best signal along with a marked improvement in the peak shape was observed for darifenacin and darifenacin-d4.

Short length columns, such as Symmetry Shield RP18 $(50 \times 2.1 \mathrm{~mm}, 3.5 \mu \mathrm{m})$, Inertsil ODS-2V $(50 \times 4.6 \mathrm{~mm}$, $5 \mu \mathrm{m})$, Hypurity C18 $(50 \times 4.6 \mathrm{~mm}, 5 \mu \mathrm{m})$, and Hypurity Advance $(50 \times 4.0 \mathrm{~mm}, 5 \mu \mathrm{m})$, were tried during the method development. Symmetry Shield RP18 column gave a relatively good peak shape, but the response was low. Using Hypurity C18 column poor chromatography was observed. The best signal was obtained using the Zorbax, SB C18, $4.6 \times 75 \mathrm{~mm}, 3.5 \mu \mathrm{m}$ column. It gave satisfactory peak shapes for both darifenacin and darifenacin-d4. Flow rate of $1.0 \mathrm{~mL} / \mathrm{min}$ without splitter was utilized and reduced the run time to 3.0 minutes for both drug and IS were eluted with shorter time at $1.3 \mathrm{~min}$. For an LC-MS/MS analysis, utilization of stable isotope-labeled or suitable analog drugs as an internal standard proves helpful when a significant matrix effect is possible. In our case, darifenacin-d4 was found to be best for the present purpose. The column oven temperature was kept at a constant temperature of about $40^{\circ} \mathrm{C}$. Injection volume of $10 \mu \mathrm{L}$ sample is adjusted for better ionization and chromatography.

3.4. Extraction Optimization. Prior to load the sample for LC injection, the coextracted proteins should be removed from the prepared solution. For this purpose, initially we tested with different extraction procedures like PPT (protein precipitation), LLE (liquid liquid extraction), and SPE (solid phase extraction). We found ion suppression effect in protein precipitation method for drug and internal standard. Further, we tried with SPE and LLE. Out of all we observed that LLE is suitable for extraction of drug and IS. We tried with several organic solvents (ethyl acetate, chloroform, n-hexane, dichloromethane, and methyl tertiary butyl ether) individually as well with combinations in LLE to extract analyte from the plasma sample. In our case methyl tertiary butyl ether: dichloromethane $(80: 20)$ combination served as good extraction solvent. Auto sampler wash is optimized as $80 \%$ methanol. High recovery and selectivity were observed in the Liquid-Liquid extraction method.
These optimized detection parameters, chromatographic conditions and extraction procedure resulted in reduced analysis time with accurate and precise detection of darifenacin in rat plasma.

3.5. Method Validation. A thorough and complete method validation of darifenacin in rat plasma was done following US FDA guidelines [21, 22]. The method was validated for system suitability, carryover, linearity, selectivity, precision and accuracy, sensitivity, matrix effect, recovery, dilution integrity, reinjection reproducibility, and stability.

3.6. System Suitability and Autosampler Carryover. Throughout the method validation, the \%CV of the system suitability was observed below $4.0 \%$ at the retention time of darifenacin and the IS. Carryover evaluation was performed in each analytical run to ensure that it does not affect the accuracy and the precision of the proposed method. There was a negligible carryover ( $\leq 5 \%$ of the LLOQ response) observed during autosampler carryover experiment. No enhancement in the response was observed in double blank after subsequent injection of the highest calibration standard (aqueous and extracted) at the retention time of analyte and IS.

\subsection{Linearity and Lower Limit of Quantification (LLOQ).} The calibration curves were linear over the concentration range of $10.00-20000.00 \mathrm{pg} / \mathrm{mL}$ with the correlation coefficient $r \geq 0.9950$ for darifenacin.

3.8. Precision and Accuracy. The accuracy and precision (\%CV) observed for the calibration curve standards ranged from 96.80 to $104.30 \%$ and 0.79 to $2.32 \%$ for darifenacin. The lowest concentration (LLOQ) in the standard curve for darifenacin was measured at a signal-to-noise ratio $(\mathrm{S} / \mathrm{N})$ of $\geq 30$. The intra- and inter-batch precision and accuracy were established from validation runs performed at HQC, MQC, LQC, and LLOQ QC levels (Table 2). The intra- and inter-batch precision ranged from 1.86 to $6.87 \%$ and 3.19 to $5.19 \%$, respectively, for darifenacin. The accuracy values were within 96.80 to $104.30 \%$ and 99.37 to $107.92 \%$ for the analyte in intra- and inter-batches.

3.9. Selectivity. To establish the selectivity of the method for interference due to endogenous plasma components from haemolysed, lipidemic, heparinized, and K2 EDTA blank plasmas, the \% change in the area ratio (analyte/IS) at LLOQ level was within $4-6 \%$, while the precision (\%CV) in their measurement varied from 2.0 to 4.5 .

The extraction procedure together with mass detection gave very good selectivity for the analysis of drug and IS in the blank plasma. No endogenous interferences were found at the retention times of analyte and IS.

3.10. Matrix Effect. Matrix effect may be defined as a composite of some undesirable effects that originate from a biological matrix. These components may result in ion suppression/enhancement, decrease/increase in sensitivity of analytes over a period of time, increased baseline, 
TABle 2: Precision and accuracy (analysis with spiked plasma samples at four different concentrations).

\begin{tabular}{lcccc}
\hline $\begin{array}{l}\text { Spiked plasma concentration } \\
(\mathrm{pg} / \mathrm{mL})\end{array}$ & $\begin{array}{c}\text { Within-run } n=6 \\
\text { Accuracy } \\
(\text { Mean } \pm \text { S.D. })\end{array}$ & $\begin{array}{c}\text { Precision } \\
(\text { CV\%) }\end{array}$ & $\begin{array}{c}\text { Between-run } n=36 \\
\text { Accuracy } \\
\text { (Mean } \pm \text { S.D. })\end{array}$ & $\begin{array}{c}\text { Precision } \\
(\text { CV\%) }\end{array}$ \\
\hline 10.00 & $11.14 \pm 0.77$ & 6.87 & $10.79 \pm 0.56$ & 5.19 \\
30.00 & $29.93 \pm 0.93$ & 3.10 & $29.81 \pm 0.99$ & 3.32 \\
9000.00 & $9042.49 \pm 167.97$ & 1.86 & $9090.34 \pm 289.83$ & 3.19 \\
18000.00 & $18457.37 \pm 253.95$ & 1.38 & $18125.79 \pm 674.07$ & 3.72 \\
\hline
\end{tabular}

TABLE 3: Stability of darifenacin in rat plasma samples.

\begin{tabular}{|c|c|c|c|c|c|c|c|c|}
\hline \multirow[b]{2}{*}{$\begin{array}{l}\text { Spiked plasma } \\
\text { concentration } \\
(\mathrm{pg} / \mathrm{mL})\end{array}$} & \multicolumn{2}{|c|}{$\begin{array}{l}\text { Bench-top stability } \\
30 \mathrm{hr}\end{array}$} & \multicolumn{2}{|c|}{$\begin{array}{c}\text { Autosampler stability } \\
65 \mathrm{hr}\end{array}$} & \multicolumn{2}{|c|}{$\begin{array}{l}\text { Long term stability } \\
55 \text { Days }\end{array}$} & \multicolumn{2}{|c|}{$\begin{array}{c}\text { Freeze and thaw stability } \\
\text { Cycle } 3(48 \mathrm{hr})\end{array}$} \\
\hline & $\begin{array}{c}\text { Concentration } \\
\text { measured }(n=6) \\
(\mathrm{pg} / \mathrm{mL}) \\
(\text { mean } \pm \text { S.D })\end{array}$ & $\begin{array}{c}\text { CV } \\
(\%) \\
(n=6)\end{array}$ & $\begin{array}{c}\text { Concentration } \\
\text { measured }(n=6) \\
(\mathrm{pg} / \mathrm{mL}) \\
(\text { mean } \pm \text { S.D })\end{array}$ & $\begin{array}{c}\text { CV } \\
(\%) \\
(n=6)\end{array}$ & $\begin{array}{c}\text { Concentration } \\
\text { measured }(n=6) \\
(\mathrm{pg} / \mathrm{mL}) \\
(\text { mean } \pm \text { S.D })\end{array}$ & $\begin{array}{c}\text { CV } \\
(\%) \\
(n=6)\end{array}$ & $\begin{array}{c}\text { Concentration } \\
\text { measured }(n=6) \\
(\mathrm{pg} / \mathrm{mL}) \\
(\text { mean } \pm \text { S.D })\end{array}$ & $\begin{array}{c}\text { CV } \\
(\%) \\
(n=6)\end{array}$ \\
\hline 30.00 & $29.60 \pm 0.81$ & 2.70 & $29.80 \pm 0.40$ & 1.34 & $28.90 \pm 0.60$ & 2.08 & $29.50 \pm 0.80$ & 2.71 \\
\hline 18000.00 & $17889.00 \pm 120.50$ & 0.67 & $17923.00 \pm 118.50$ & 0.66 & $17863.00 \pm 161.80$ & 0.90 & $17943.00 \pm 135.20$ & 0.75 \\
\hline
\end{tabular}

imprecision of data, drift in retention time, and distortion or tailing of a chromatographic output. Result of postcolumn infusion experiment indicates no ion suppression or enhancement at the retention time of analyte and IS as evident from the flat baseline. There is no ion suppression and enhancement observed at retention time of analyte and IS.

3.11. Recovery. The relative recovery and process efficiency data at LQC, MQC, and HQC levels were observed as 93.40, 95.70 , and 94.70 . The recovery for IS in rat plasma was 93.9\%.

\subsection{Stability}

3.12.1. Stock Solution Stability. Stock solution stability was performed to check stability of darifenacin and darifenacinD4 in stock solutions prepared in methanol and stored at 2$8^{\circ} \mathrm{C}$ in a refrigerator. The freshly prepared stock solutions were compared with stock solutions prepared before 15 days. The \% change for darifenacin and darifenacin D4 were $-0.04 \%$ and $0.03 \%$, respectively, indicating that stock solutions were stable for at least 15 days.

3.12.2. Stability in Plasma. Bench top and autosampler stability for darifenacin was investigated at LQC and HQC levels. The results revealed that darifenacin was stable in plasma for at least $30 \mathrm{~h}$ at room temperature, and $65 \mathrm{~h}$ in an auto sampler. It was confirmed that repeated freezing and thawing (three cycles) of plasma samples spiked with darifenacin at LQC and HQC levels did not affect their stability. The long-term stability results also indicated that darifenacin was stable in a matrix up to 55 days at a storage temperature of $-30^{\circ} \mathrm{C}$. The results obtained from all these stability studies are tabulated in Table 3.
TABLE 4: Mean pharmacokinetic parameters of darifenacin in rat plasma after intravenous administration of $0.27 \mathrm{mg} / 200 \mathrm{~g}$ male rat.

\begin{tabular}{lc}
\hline Pharmacokinetic parameter & Darifenacin \\
\hline $\mathrm{AUC}_{0-t}(\mathrm{pg} \cdot \mathrm{h} / \mathrm{mL})$ & 28961.88 \\
$C_{\max }(\mathrm{pg} / \mathrm{mL})$ & 9109 \\
$\mathrm{AUC}_{0-\infty}(\mathrm{pg} \cdot \mathrm{h} / \mathrm{mL})$ & 29847.67 \\
$\mathrm{Kel}\left(\mathrm{h}^{-1}\right)$ & 0.14225 \\
$T_{\max }(\mathrm{h})$ & 0.25 \\
\hline
\end{tabular}

$\mathrm{AUC}_{0-\infty}$ : area under the curve extrapolated to infinity, $\mathrm{AUC}_{0-t}$ : area under the curve up to the last sampling time, $C_{\max }$ : the maximum plasma concentration, $T_{\max }$ : the time to reach peak concentration, Kel: the apparent elimination rate constant.

3.13. Ruggedness. The results of ruggedness study for darifenacin were well within the acceptance limit of $15 \%$ in precision and $85.0-115.0 \%$ in mean accuracy. The precision and accuracy values for both experiments at LLOQ, LQC, MQC, and HQC levels for darifenacin ranged from 1.6 to $4.9 \%$ and 98.4 to $107.2 \%$, respectively.

3.14. Dilution Integrity. The dilution integrity experiment was performed with an aim to validate the dilution test to be carried out on higher analyte concentration above the upper limit of quantification (ULOQ), which may be encountered during real subject sample analysis. The precision and accuracy values for $1 / 4$ th and $1 / 10$ th dilution ranged from $4.9 \%$ and $102.8 \%$ for darifenacin.

3.15. Application of the Method. The validated method has been successfully applied to quantify darifenacin concentrations in to a single dose $(0.27 \mathrm{mg} / 200 \mathrm{~g})$ in rats. Male Sprague-Dawley rats were obtained from Bioneeds, Bangalore. After i.v. administration of drug via left femoral vein $0.2 \mathrm{~mL}$ of blood samples for analytical determinations 


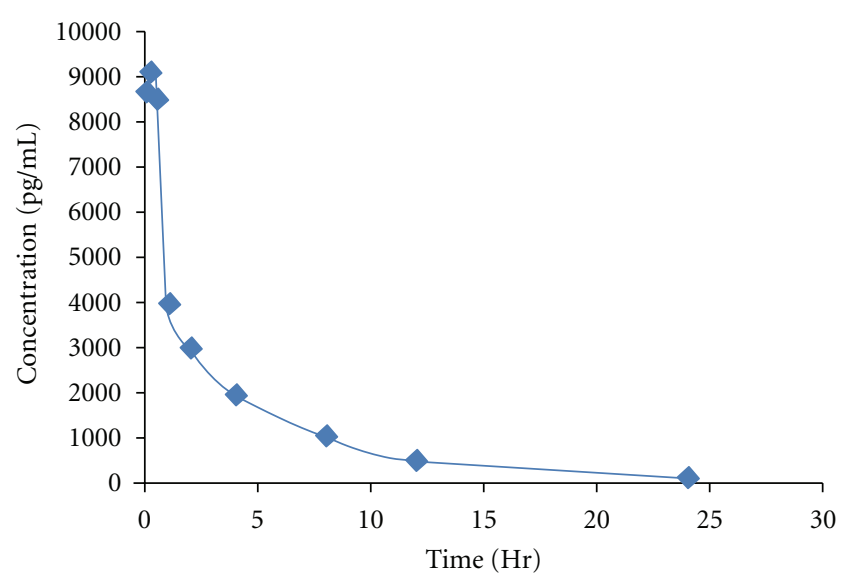

Figure 5: Mean plasma concentrations versus time graph of darifenacin after intravenous administration of $0.27 \mathrm{mg} / 200 \mathrm{~g}$ in male rat.

were collected via the right femoral vein at specific time intervals for $24 \mathrm{~h}$. Plasma samples were stored at $-30^{\circ} \mathrm{C}$ until analysis. The study was carried out after approval from an independent animal ethics committee. The pharmacokinetic parameters evaluated were Cmax (maximum observed drug concentration during the study), $\mathrm{AUC}_{0-24}$ (area under the plasma concentration-time curve measured 24 hours, using the trapezoidal rule), $T_{\max }$ (time to observe maximum drug concentration), Kel (apparent first order terminal rate constant calculated from a semi-log plot of the plasma concentration versus time curve, using the method of least square regression), and $T_{1 / 2}$ (terminal half-life as determined by quotient $0.693 / \mathrm{Kel})$. Pharmacokinetic details were shown in Table 4. The mean concentration versus time profile of darifenacin in rat plasma is shown in Figure 5.

\section{Conclusion}

The proposed method exhibited excellent performance in terms of sensitivity, selectivity, ruggedness, and efficiency (3.0 min per sample) due to cleaner extracts, with simplicity of sample preparation. This method was successfully applied in pharmacokinetics of rat plasma.

\section{Acknowledgments}

The authors are grateful to the Indian Institute of chemical technology, Hyderabad for Literature survey and Acron Accunova, Manipal, India for their laboratory facility of this research work.

\section{References}

[1] A. Tomillero and M. A. Moral, "Gateways to clinical trials," Methods and Findings in Experimental and Clinical Pharmacology, vol. 32, no. 10, pp. 749-773, 2010.

[2] A. Skerjanec, "The clinical pharmacokinetics of darifenacin," Clinical Pharmacokinetics, vol. 45, no. 4, pp. 325-350, 2006.

[3] K. C. Beaumont, N. J. Cussans, D. J. Nichols, and D. A. Smith, "Pharmacokinetics and metabolism of darifenacin in the mouse, rat, dog and man," Xenobiotica, vol. 28, no. 1, pp. 63-75, 1998.

[4] S. Hill, M. Elhilali, R. J. Millard et al., "Long-term darifenacin treatment for overactive bladder in patients aged 65 years and older: analysis of results from a 2-year, open-label extension study," Current Medical Research and Opinion, vol. 23, no. 11, pp. 2697-2704, 2007.

[5] T. Kerbusch, P. A. Milligan, and M. O. Karlsson, "Assessment of the relative in vivo potency of the hydroxylated metabolite of darifenacin in its ability to decrease salivary flow using pooled population pharmacokinetic-pharmacodynamic data," British Journal of Clinical Pharmacology, vol. 57, no. 2, pp. 170-180, 2004.

[6] D. Sridharan, U. A. Thenmozhi, L. P. Kumar, A. D. Chintalapati, M. V. Ramanaiah, and Y. Phanikishore, "Development and validation of UV spectrophotometric method of darifenacin hydrobromide in bulk and tablet dosage form," Asian Journal of Pharmaceutical Analysis, vol. 1, no. 3, pp. 43-45, 2011.

[7] P. S. Sai Praveen, V. Jagathi, G. Devala Rao, and G. Sudhakar Saibabu, "Visible spectrophotometric methods for the determination of darifenacin," Research Journal of Pharmaceutical, Biological and Chemical Sciences, vol. 1, no. 2, pp. 254-257, 2010.

[8] P. Sai Praveen, M. Shaiba, A. Swetha, A. Aruna Jyothi, and A. Nishat, "Spectrophotometric methods for the determination of darifenacin," Research Journal of Pharmaceutical, Biological and Chemical Sciences, vol. 1, no. 4, pp. 350-353, 2010.

[9] P. Sai Praveen, G. Devala Rao, V. Jagathi, M. Shaiba, and C. H. Manohar Babu, "Spectroflourimetric method for the estimation of darifenacin in pure and pharmaceutical formulations," Asian Journal of Pharmaceutical and Clinical Research, vol. 4, no. 1, pp. 44-46, 2011.

[10] S. Kathirvel, S. V. Satyanarayana, and G. Devalarao, "Densitometric evaluation of stability-indicating HPTLC Method for the analysis of darifenacin hydrobromide in bulk and in tablet dosage form," Journal of Liquid Chromatography and Related Technologies, vol. 35, no. 2, pp. 280-293, 2012.

[11] V. Rajesh, K. Rajendra Prasad, A. Suneetha, and S. Kathirvel, "Development and validation of Rp-Hplc method for estimation of darifenacin hydrobromide in bulk and tablet dosage form," Asian Journal of Research in Chemistry, vol. 4, no. 6, 2011.

[12] P. Radhakrishnanand, D. V. Subba Rao, and V. Himabindu, "A validated LC method for determination of the enantiomeric purity of darifenacin in bulk drug and extended release tablets," Chromatographia, vol. 68, no. 11-12, pp. 1059-1062, 2008.

[13] L. Z. Meneghini, C. Junqueira, S. A. Andrade et al., "Chemometric evaluation of darifenacin hydrobromide using a stability-indicating reversed-phase LC method," Journal of Liquid Chromatography and Related Technologies, vol. 34, no. 18, pp. 2169-2184, 2011.

[14] A. Thenmozhi, Umarani, and D. Sridharan, "A stability indicating HPLC method for the estimation of darifenacin hydrobromide in pure and tablet dosage form," Asian Journal of Biochemical and Pharmaceutical Research, vol. 3, no. 1, pp. 48-61, 2011.

[15] S. Thomas, S. K. Paul, S. Shandilya et al., "Identification and structural elucidation of two process impurities and stress degradants in darifenacin hydrobromide active pharmaceutical ingredient by LC-ESI/MSn," Analyst, vol. 137, no. 15, pp. 3571-3582, 2012.

[16] A. Yoshida, S. Maruyama, D. Fukumoto, H. Tsukada, Y. Ito, and S. Yamada, "Noninvasive evaluation of brain muscarinic 
receptor occupancy of oxybutynin, darifenacin and imidafenacin in rats by positron emission tomography," Life Sciences, vol. 87, no. 5-6, pp. 175-180, 2010.

[17] S. Maruyama, H. Tsukada, S. Nishiyama et al., "In vivo quantitative autoradiographic analysis of brain muscarinic receptor occupancy by antimuscarinic agents for overactive bladder treatment," Journal of Pharmacology and Experimental Therapeutics, vol. 325, no. 3, pp. 774-781, 2008.

[18] Y. Ito, L. Oyunzul, M. Seki, T. Fujino, M. Matsui, and S. Yamada, "Quantitative analysis of the loss of muscarinic receptors in various peripheral tissues in M1-M5 receptor single knockout mice," British Journal of Pharmacology, vol. 156, no. 7, pp. 1147-1153, 2009.

[19] Y. Masuda, N. Kanayama, S. Manita, S. Ohmori, and T. Ooie, "Development and validation of bioanalytical methods for Imidafenacin (KRP-197/ONO-8025) and its metabolites in human plasma by liquid chromatography-tandem mass spectrometry," Journal of Chromatography B, vol. 853, no. 12, pp. 70-79, 2007.

[20] B. Kaye, W. J. Herron, P. V. Macrae et al., "Rapid, solid phase extraction technique for the high-throughput assay of darifenacin in human plasma," Analytical Chemistry, vol. 68, no. 9 , pp. 1658-1660, 1996.

[21] Center for Biologics Evaluation and Research (CBER), Guidance for Industry: Bioanalytical Method Validation, U.S. Department of Health and Human Services, Food and Drug Administration, Center for Drug Evaluation and Research (CDER), 2001.

[22] Centre for Drug Evaluation and Research (CDER), Guidance for Industry Food- Effect Bio Availability and Fed Bio Equivalence Studies, U.S. Department of Health and Human services Food and Drug Administration, Centre for Drug Evaluation and Research (CDER), 2002. 


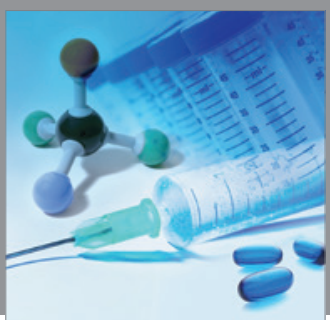

International Journal of

Medicinal Chemistry

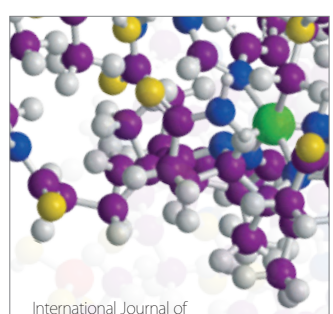

Carbohydrate Chemistry

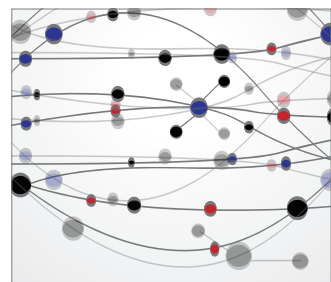

The Scientific World Journal
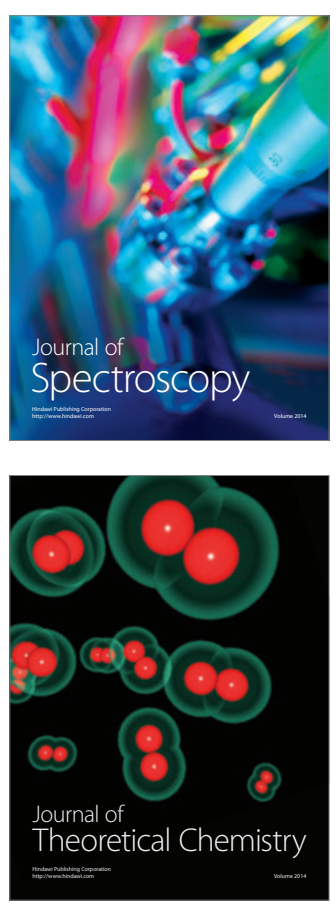
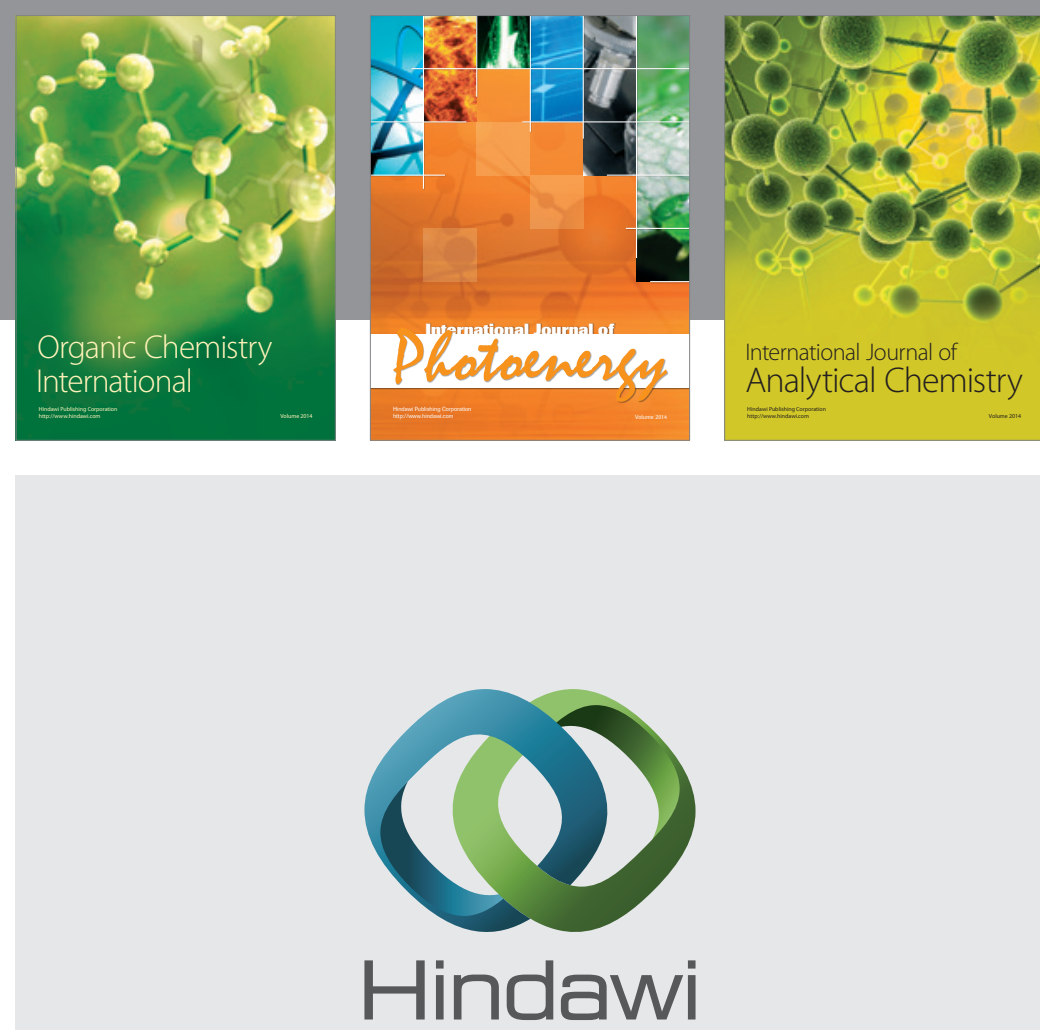

Submit your manuscripts at

http://www.hindawi.com
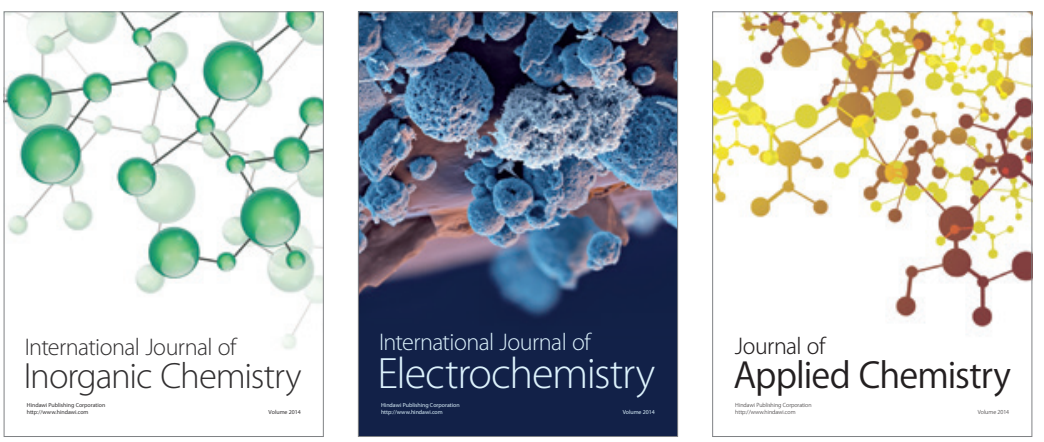

Journal of

Applied Chemistry
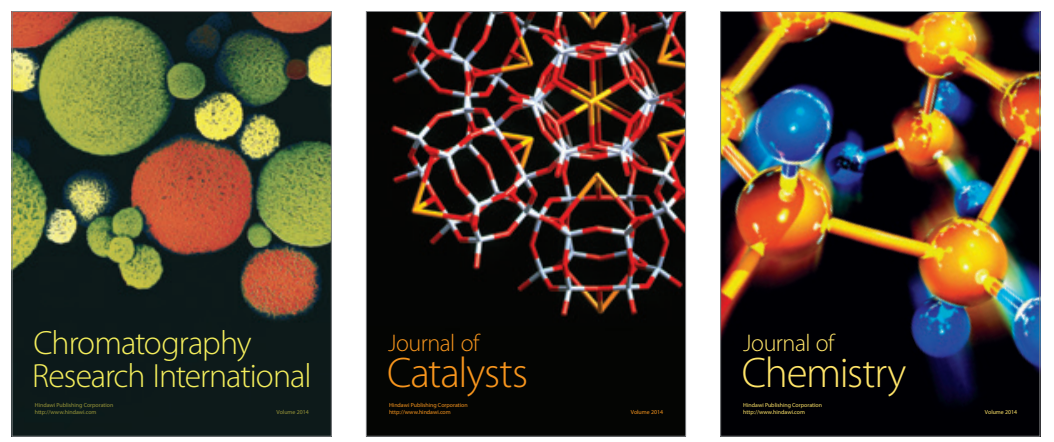
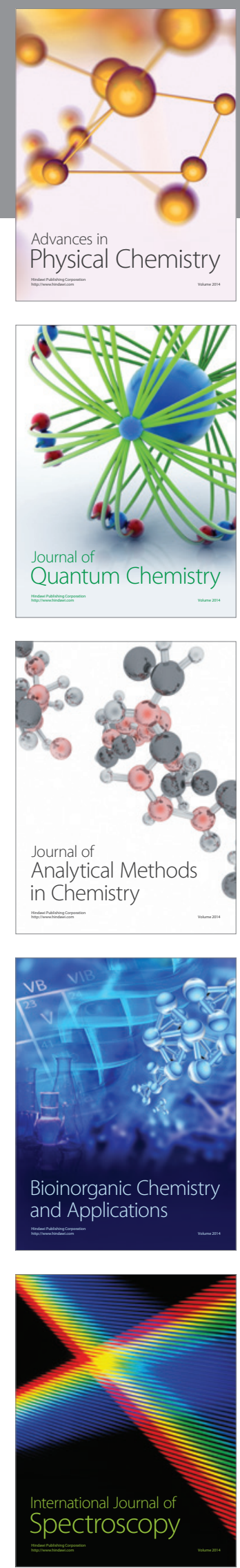